ఠ

\title{
A significant response to a combination of trastuzumab and vinorelbine in HER2-negative metastatic breast cancer with HER2 V777L mutation
}

This article was published in the following Dove Medical Press journal:

OncoTargets and Therapy

\author{
Lei Lei ${ }^{1,2, *}$ \\ Wei-Wu Ye ${ }^{2, *}$ \\ Lin-Feng Zheng ${ }^{3}$ \\ Ping Huang ${ }^{2}$ \\ Lei $\mathrm{Shi}^{2}$ \\ Jian Huang ${ }^{2}$ \\ Ya-Bing Zheng ${ }^{2}$ \\ Zhan-Hong Chen ${ }^{2}$ \\ Xiao-Jia Wang ${ }^{2}$ \\ Xian Wang' \\ 'Department of Medical Oncology, \\ Sir Run Run Shaw Hospital, Zhejiang \\ University School of Medicine, \\ Hangzhou, Zhejiang Province, \\ China; ${ }^{2}$ Department of Medical \\ Oncology, Zhejiang Cancer Hospital, \\ Hangzhou, Zhejiang Province, China; \\ ${ }^{3}$ Department of Pathology, Zhejiang \\ Cancer Hospital, Hangzhou, Zhejiang \\ Province, China
}

*These authors contributed equally to this work
Correspondence: Xian Wang Department of Medical Oncology, Sir Run Run Shaw Hospital, Zhejiang University School of Medicine, Hangzhou 310020, Zhejiang, China Tel +86057186006366

Email wangxII8@zju.edu.cn

\begin{abstract}
Metastatic breast cancer (MBC) is the most life-threatening disease in women worldwide. HER2-mutated breast carcinoma has been reported to benefit from HER2-targeted tyrosine kinase inhibitors recently. Here, we presented a heavy pretreated and harbored HER2 V777L mutation de novo stage IV Luminal B (HER2 unamplified) breast cancer patient who achieved an unexpected good response to trastuzumab combined with vinorelbine therapy. Although HER2-unamplified MBC patients do not regularly benefit from anti-HER2 target therapy, HER2 V777L mutation detected by next-generation sequencing from ctDNA may present as a predictive biomarker for anti-HER2-based strategy therapy in HER2-negative MBC patients.
\end{abstract}

Keywords: metastatic breast cancer, HER2 gene mutation, trastuzumab

\section{Introduction}

In China, the most recent official data indicate continued increases in breast carcinoma incidence and associated mortality rates. ${ }^{1}$ Although the introduction of trastuzumab, a therapeutic monoclonal antibody specific for the human epidermal growth factor receptor 2 (HER2), has revolutionized the management of HER2-positive breast cancers, ${ }^{2}$ many patients present with HER2-negative tumors that are unresponsive to HER2-targeted therapies. All protocols in the present study were approved by the Human Clinical and Research Ethics Committee of the Zhejiang Cancer Hospital (Hangzhou, China). The patient provided written informed consent for the publication of case details and any accompanying images.

According to a previous report, about $2 \%$ of the patients with HER2-negative breast cancer harbor a somatic mutation in HER2, and such mutation was found to be associated with poor survival. ${ }^{3}$ In addition, a HER2 somatic mutation is considered a potential alternative pathway to HER2 activation; therefore, such tumors may be sensitive to anti-HER2 therapy. ${ }^{4}$ Here, we present a case of a patient with metastatic breast cancer (MBC) who harbored a HER2 V777L mutation despite a lack of HER2 amplification (via fluorescent in situ hybridization, FISH) from the tumor tissue. This patient achieved a significant clinical response to a combination chemotherapy regimen that included trastuzumab.

\section{Case report}

A 47-year-old Chinese woman was initially diagnosed with de novo stage IV breast cancer at a local hospital in September 2016. Breast ultrasonography located a primary 
lesion measuring $44 \times 40 \times 29 \mathrm{~mm}$ behind the left nipple, and abdominal computed tomography (CT) detected multiple lesions in both lobes of the liver. A core needle biopsy of the left breast mass revealed invasive ductal carcinoma (IDC). An immunohistochemical (IHC) study revealed an estrogen receptor-positive $(\mathrm{ER}+)$ frequency of $40 \%$, progesterone receptor-positive $(\mathrm{PR}+)$ frequency of $20 \%$, Ki-67 index frequency of $15 \%$, and HER2 negativity (HER2-). Pathologic analysis of a liver tumor biopsy revealed metastatic IDC, with an IHC status of $70 \% \mathrm{ER}+, 70 \% \mathrm{PR}+, 15 \% \mathrm{Ki}-67+$, and HER2-. Although she subsequently received several lines of chemotherapy and hormonal therapy (fulvestrant; docetaxel+ epirubicin+cyclophosphamide (TEC); paclitaxel; transcatheter arterial chemoembolization [TACE]; anastrozole and capecitabine), the tumors progressed rapidly.

On September 11, 2017, she visited Zhejiang Cancer Hospital with the complaint of a gradually enlarged and painful mass in her left breast and was determined to have an Eastern Cooperative Oncology Group performance score of 1 . She had experienced regular menstrual cycles since undergoing menarche at 13 years of age and gave birth to her first child, who was breastfed, at 26 years of age. She entered menopause at 47 years of age. She had no history of oral contraceptive use, no family history of breast cancer, no psychosocial history, and no co-morbidities. Figure 1 summarizes the clinical course of this patient, who provided informed consent for the publication of these case details.

After she arrived at our hospital, physical and imaging examinations revealed a bulky mass in the left breast, multiple enlarged lymph nodes in the left axilla, and multiple massive tumors in both lobes of the liver (Figures 2A and $3 \mathrm{~A}-\mathrm{C}$ ). A core needle biopsy of the primary lesion was obtained for IHC and next-generation sequencing (NGS) analyses, which sequenced the entire coding regions of 365 cancer-related genes and 47 introns of 25 genes frequently rearranged in cancer (Table 1). The pathologic analysis revealed IDC, with an IHC status of HER2 2+, 65\% ER+, $5 \% \mathrm{PR}+$, and 40\% Ki-67+. FISH indicated HER2- (HER2 signals $=3.43$, CEP17 signals $=2.67$, HER2 $/$ CEP $17=1.29$, and chromosome $17=$ diploid) (Figure 4A-C). A pathologic review of the liver lesion prior to initial treatment indicated a metastasis of breast origin and the following IHC status: 70\% ER+, 70\% PR+, 15\% Ki-67+, and HER2-(Figure 4D and $\mathrm{E}$ ). Consistent with the prior IHC and FISH test results for the lesions, NGS of the left breast tumor did not detect ERBB2 amplification but rather identified a V777L mutation in HER2 at an allelic frequency of $40.90 \%$. This mutation was also detected in circulating tumor DNA (ctDNA) at an allelic

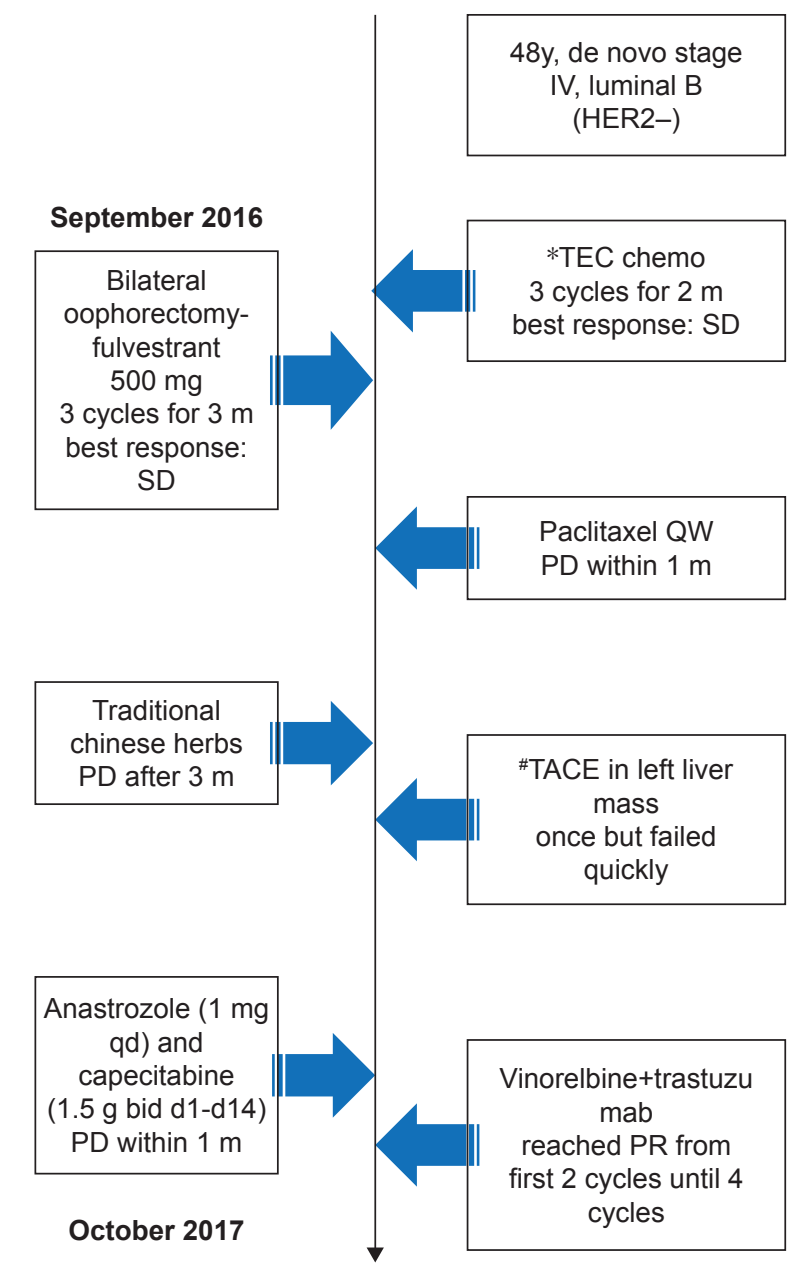

Figure I Timeline of the present case.

Abbreviations: bid, twice a day; $m$, month; PR, partial response; qd, once a day; TEC, docetaxel + epirubicin + cyclophosphamide; TACE, transcatheter arterial chemoembolization; $y$, years; HER2, human epidermal growth factor receptor 2; $\mathrm{SD}$, stable disease; PD, progression of disease.

frequency of $33.85 \%$ (Table 1 and Figure 5). A mutation in TP53 (G245V: 70.6\% in breast tumor, $41.51 \%$ in ctDNA) was also identified.

Following a multidisciplinary team conference, a treatment regimen comprising vinorelbine $\left(25 \mathrm{mg} / \mathrm{m}^{2}\right.$ on days 1 and 8 , every 3 weeks [Q3W]) plus trastuzumab (6 mg/kg, $8 \mathrm{mg} / \mathrm{kg}$ loading dose, $\mathrm{q} 3 \mathrm{w}$ ) was administered, which quickly relieved the pain in the left breast (Figure 2). A grade 1/2 gastrointestinal reaction and hematologic toxicity were observed during 4 cycles of this regimen. CT scans showed significant shrinkage of all lesions after the first 2 cycles of treatment, although heterogeneous responses were observed in the right and left liver lobes (Figure 3). Ultimately, the patient achieved a partial response (PR). Although the National Comprehensive Cancer Network guidelines for therapeutic efficacy evaluation do not recommend regular biological tumor marker detection, we observed remarkable declines in 

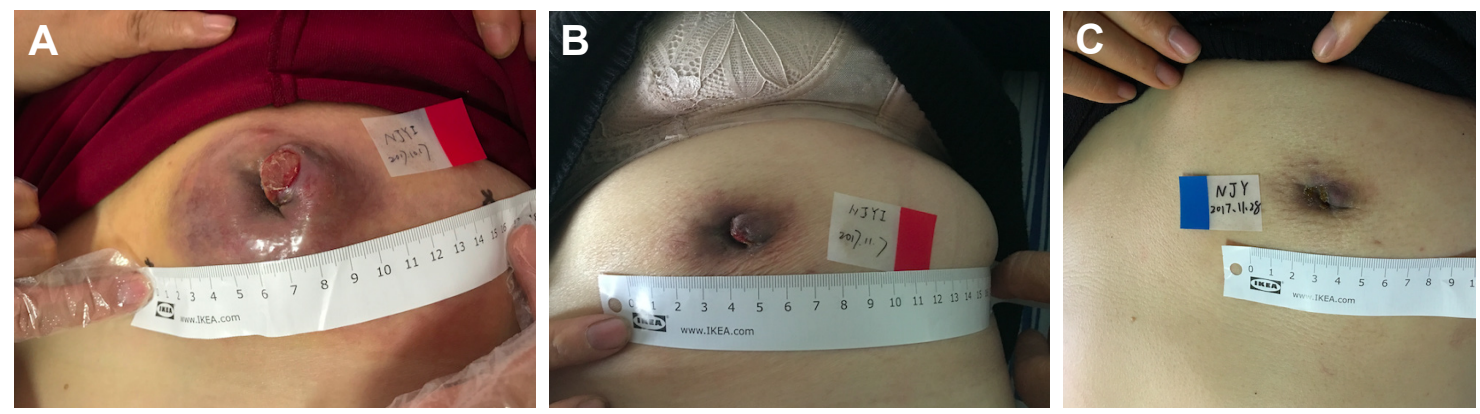

Figure 2 Photographs of the primary lesion in the patient's left breast.

Notes: (A) Before VT therapy. (B) After 2 cycles of VT therapy. (C) After 4 cycles of VT therapy

Abbreviation: VT, vinorelbine + trastuzumab.
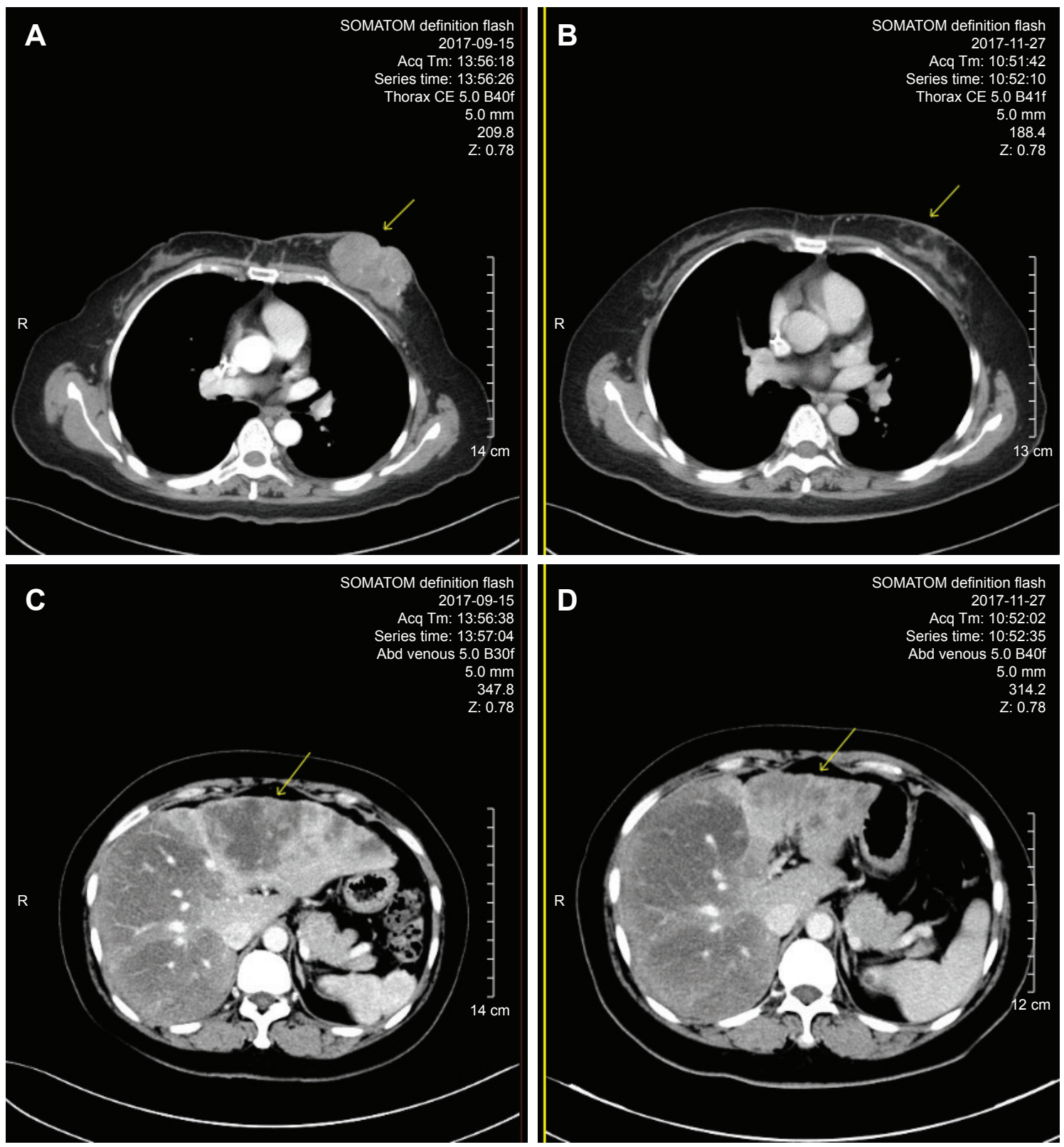

Figure 3 Comparison of computed tomography scans before and after 2 cycles of vinorelbine + trastuzumab regimen.

Note: (A and B) Left breast lesion before and after treatment, respectively; (C and $\mathbf{D})$ left liver lesions before and after treatment, respectively. 
Table I Detection of HER2-related biomarkers before treatment

\begin{tabular}{|l|l|l|}
\hline Tumor source & $\begin{array}{l}\text { HER2 } \\
\text { amplification }\end{array}$ & $\begin{array}{l}\text { HER2 V777L } \\
\text { (AF, \%) }\end{array}$ \\
\hline Left breast lesion & Negative & 40.9 \\
\hline Liver lesion & Negative & - \\
\hline ctDNA & Negative & 33.85 \\
\hline
\end{tabular}

Abbreviations: AF, allelic frequency; ctDNA, circulating tumor DNA; HER2, human epidermal growth factor receptor 2 .

the patient's serum carbohydrate antigen 153 (from 180.34 to $6.9 \mathrm{ng} / \mathrm{mL}$ ) and carcinoembryonic antigen levels (from $3,228.7$ to $330.5 \mathrm{U} / \mathrm{mL}$ ).

\section{Discussion}

Advances in chemotherapy and endocrine therapy have yielded impressive improvements in the clinical outcomes of patients with ER+, HER2- breast cancer. However, some de novo MBCs inevitably exhibit metastatic multidrug resistance, ${ }^{5}$ and effective strategies for these refractory cases are urgently needed.

Since its introduction almost 2 decades ago, anti-HER2 therapy has successfully prolonged the survival of many patients with HER2-positive MBC. ${ }^{6}$ Although somatic HER 2 mutation has been suggested as a potential mechanism underlying resistance to trastuzumab, ${ }^{7}$ patients harboring unamplified or mutated HER2 may also respond to antiHER2 agents. ${ }^{4,8}$ In our case, NGS analyses of the breast tumor and ctDNA consistently revealed the presence of the HER2 V777L mutation prior to anti-HER2 therapy. The significant clinical response of this previously heavily treated and refractory HER2-mutant, non-HER2-amplified MBC to trastuzumab-based mono-chemotherapy was thus highly interesting.
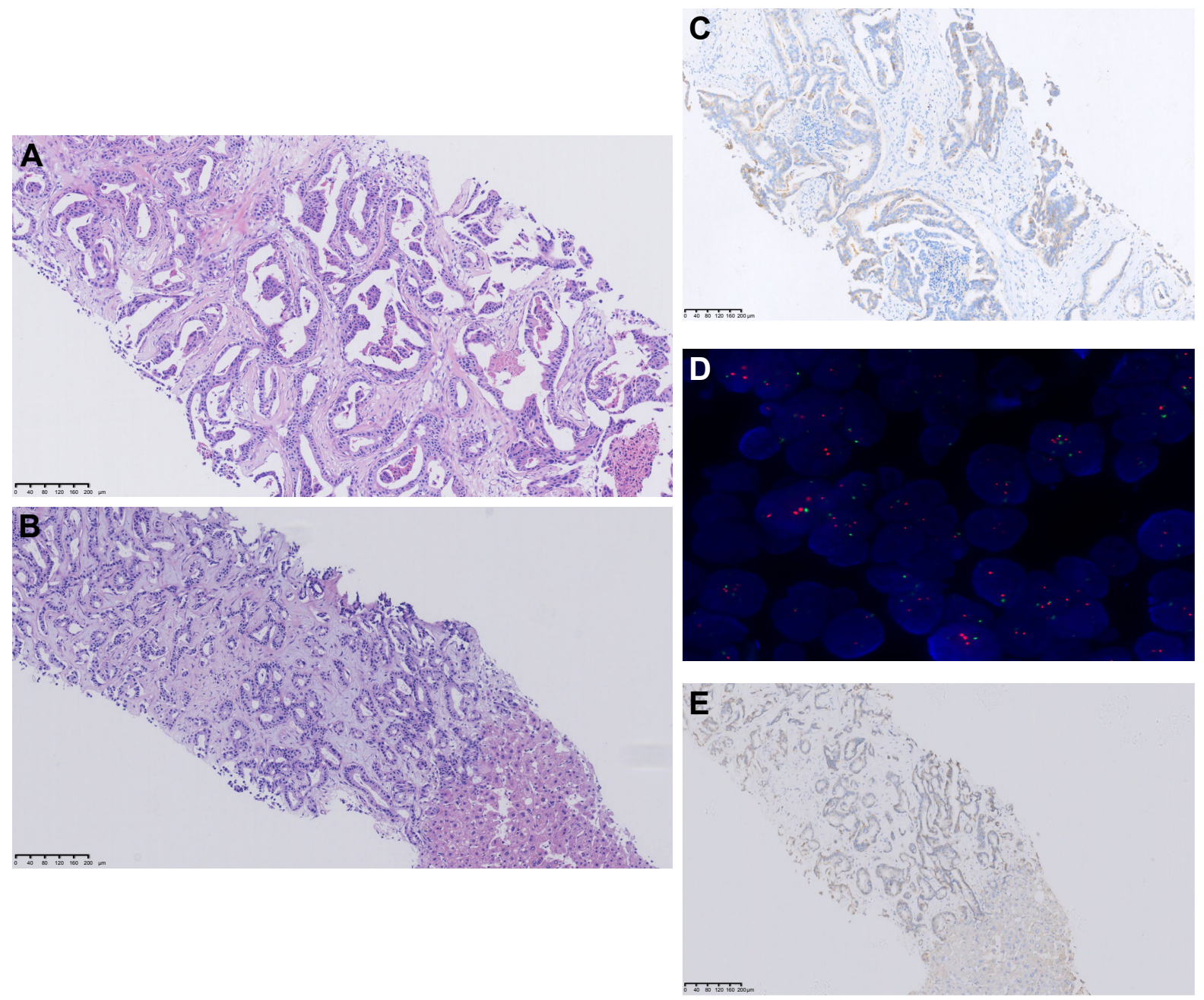

Figure 4 Analysis of the primary left breast and liver lesion tissue.

Notes: (A) H\&E; (C) IHC, HER2 2+; (D) FISH, HER2-; analysis of the primary breast lesion. (B) H\&E; (E) IHC, HER2-; analysis of metastatic liver lesion. H\&E stained images are depicted at $100 \times$ magnification.

Abbreviations: FISH, fluorescence in situ hybridization; HER2, human epidermal growth factor receptor 2; IHC, immunohistochemistry. 


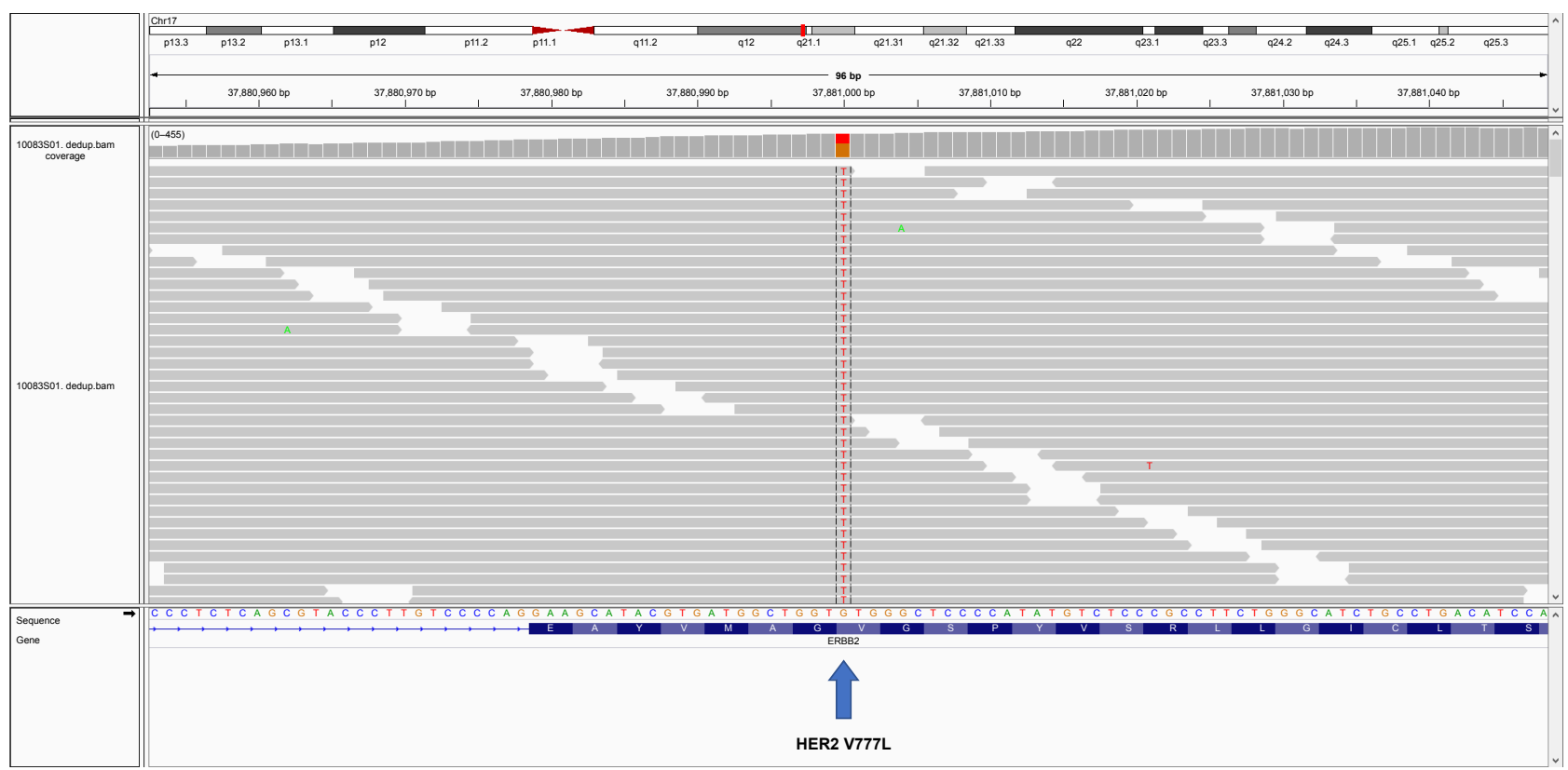

Figure 5 HER2 V777L mutation identified from ctDNA sequencing.

Note: A screenshot of the Integrative Genomics Viewer is shown.

Abbreviations: A, adenine; C, cytosine; ctDNA, circulating tumor DNA; G, guanine; HER2, human epidermal growth factor receptor 2; T, thymine.

Although reports from around the world describe overall HER 2 mutation rates of approximately $1.6 \%-9 \%$ in $\mathrm{MBC},{ }^{8-10}$ much higher rates are reported in Asian countries, with values of $16.7 \%$ in Korea and $13.3 \%$ in China. ${ }^{10}$ However, according to ctDNA data obtained at our institution, $9 \%(4 / 44)$ of the MBC patients seen between August 21, 2015, and June 12, 2018, were found to harbor the HER2 V777L mutation. This discrepancy may be attributable to differences in ethnicities or deep sequencing profiles.

Approximately two-thirds of known HER 2 mutations were detected in HER2-negative breast cancers, ${ }^{8}$ and a majority of HER2 somatic mutations drive tumorigenesis in HER2MBCs. In a recent report of a Chinese cohort of patients with MBC, 13.3\% (16/120) harbored HER2 mutations. ${ }^{10}$ Most such mutations are detected in the tyrosine kinase domain of HER2 protein, primarily in exons $19-20$. The most frequent mutation point is HER2 L755S (24\%) in exon 19, followed by HER2 V777L (13.7\%) in exon 20. Eight HER2 mutations have been proven to enhance kinase activity and tumorigenesis in preclinical studies. ${ }^{4,11}$ Additionally, several mutation carriers exhibit resistance to lapatinib but sensitivity to neratinib, which could irreversibly inhibit the HER2 pathway. ${ }^{12}$

The HER2 mutation was first reported in lung cancer, ${ }^{13}$ and subsequent preclinical studies of breast and colorectal cancer found that these mutations were responsive to antiHER2 agents, such as trastuzumab, lapatinib, and neratinib., ${ }^{4,14}$ Interestingly, a previous case of HER2-mutated inflammatory breast carcinoma responded impressively to a combination of lapatinib or trastuzumab with chemotherapy ${ }^{15}$ Recently, the HER2 V777L mutation was found to induce acquired resistance to trastuzumab in HER2-amplified breast cancer. ${ }^{16}$ According to some retrospective studies of non-MBCs, this mutation appears to correlate with worse relapse-free survival (vs wild-type HER2) among HER2-positive patients but not among HER2-negative patients. ${ }^{3,12}$ Furthermore, a recent prospective study in China reported worse progression-free survival among patients with HER2 amplification and mutation, compared to those lacking the mutation (5.0 vs 8.4 months). ${ }^{10}$ Although patients who lack HER2 amplification but harbor a mutation may remain sensitive to anti-HER2 therapy, particularly irreversible tyrosine kinase inhibitors, ${ }^{10,17}$ the prognostic value and responses of HER2-mutated MBCs lacking HER2 amplification to trastuzumab therapy have not previously been elucidated. Although a possible association between HER2 pathway resistance and HER2 amplification in MBCs has been reported, ${ }^{9,16}$ an HER2 mutation cannot be considered an HER2 resistance mechanism in these tumors. ${ }^{18}$ Accordingly, in the present case we prescribed trastuzumab together with vinorelbine as a potentially effective anti-HER2 palliative therapy regimen.

\section{Conclusion}

The relationship between the HER2 mutation status and the effects of HER2-targeted therapies in HER2-negative MBCs 
remains unclear. However, the HER2 mutation status should be considered in both HER2-amplified and non-amplified patients with MBC and particularly in heavily pretreated and refractory cases. Additionally, predictive biomarkers are needed to optimize palliative strategies for advanced and refractory breast cancers.

\section{Ethics approval and consent form}

The case reported in the study was approved by the ethics committee of Zhejiang Cancer Hospital. Consent was obtained from the patient for the use of images and medical information in this study.

\section{Acknowledgment}

This work was supported by the Zhejiang Province Medical Science Fund Project of China (2015KYB052) and the clinical research fund of Zhejiang Medical Association (2016ZYC-A06).

\section{Disclosure}

The authors report no conflicts of interest in this work.

\section{References}

1. Chen W, Zheng R, Baade PD, et al. Cancer statistics in China, 2015. CA Cancer J Clin. 2016;66:115-132. doi:10.3322/caac.21338

2. Gyawali B, Niraula S. Duration of adjuvant trastuzumab in HER2 positive breast cancer: overall and disease free survival results from meta-analyses of randomized controlled trials. Cancer Treat Rev. 2017;60:18-23. doi:10. 1016/j.ctrv.2017.08.001

3. Wang T, Xu Y, Sheng S, et al. HER2 somatic mutations are associated with poor survival in HER2-negative breast cancers. Cancer Sci. 2017; 108:671-677. doi:10.1111/cas.13182

4. Bose R, Kavuri SM, Searleman AC, et al. Activating HER2 mutations in HER2 gene amplification negative breast cancer. Cancer Discov. 2013;3:224-237. doi:10.1158/2159-8290.CD-12-0349

5. Saeki T, Tsuruo T, Sato W, Nishikawsa K. Drug resistance in chemotherapy for breast cancer. Cancer Chemother Pharmacol. 2005;56: 84-89. doi:10.1007/s00280-005-0106-4
6. Slamon DJ, Leyland-Jones B, Shak S, et al. Use of chemotherapy plus a monoclonal antibody against HER2 for metastatic breast cancer that overexpresses HER2. N Engl J Med. 2001;344:783-792. doi:10.1056/ NEJM200103153441101

7. Fang Y, Jiang Y, Wang X, Yang X, Gao Y, Wang J. Somatic mutations of the HER2 in metastatic breast cancer. Tumour Biol. 2014;35: 11851-11854. doi:10.1007/s13277-014-2414-y

8. Petrelli F, Tomasello G, Barni S, Lonati V, Passalacqua R, Ghidini M. Clinical and pathological characterization of HER2 mutations in human breast cancer: a systematic review of the literature. Breast Cancer Res Treat. 2017;166:339-349. doi:10.1007/s10549-017-4419-x

9. Park YH, Shin HT, Jung HH, et al. Role of HER2 mutations in refractory metastatic breast cancers: targeted sequencing results in patients with refractory breast cancer. Oncotarget. 2015;6:32027-32038. doi:10.18632/ oncotarget.5184

10. Yi Z, Ma F, Guan Y, et al. Impact of HER2 mutation status on personalized molecular targeted therapy in advanced breast cancers. Abstract 1039 presented at: Annual Meeting of the American Society of Clinical Oncology; June 1-5; 2018; Chicago, IL. doi:10.1200/JCO. 2018.36.15_suppl.1039

11. Wang SE, Narasanna A, Perez-Torres M, et al. HER2 kinase domain mutation results in constitutive phosphorylation and activation of HER2 and EGFR and resistance to EGFR tyrosine kinase inhibitors. Cancer Cell. 2006;10:25-38. doi:10.1016/j.ccr.2006.05.023

12. Zuo WJ, Jiang YZ, Wang YJ, et al. Dual characteristics of novel HER2 kinase domain mutations in response to HER2-targeted therapies in human breast cancer. Clin Cancer Res. 2016;22:4859-4869. doi:10.1158/1078-0432.CCR-15-3036

13. Stephens $P$, Hunter C, Bignell G, et al. Lung cancer: intragenic ERBB2 kinase mutations in tumours. Nature. 2004;431:525-526. doi:10.1038/ $431525 b$

14. Kavuri SM, Jain N, Galimi F, et al. HER2 activating mutations are targets for colorectal cancer treatment. Cancer Discov. 2015;5:832-841. doi:10.1158/2159-8290.CD-14-1211

15. Ali SM, Alpaugh RK, Downing SR, et al. Response of an ERBB2mutated inflammatory breast carcinoma to human epidermal growth factor receptor 2-targeted therapy. J Clin Oncol. 2014;32:e88-e91. doi:10.1200/JCO.2013.49.0599

16. Hirotsu Y, Nakagomi H, Amemiya K, et al. Intrinsic HER2 V777L mutation mediates resistance to trastuzumab in a breast cancer patient. Med Oncol. 2017;34:3.

17. Ma CX, Bose R, Gao F, et al. Neratinib efficacy and circulating tumor DNA detection of HER2 mutations in HER2 nonamplified metastatic breast cancer. Clin Cancer Res. 2017;23:5687-5695. doi:10.1158/ 1078-0432.CCR-17-0900

18. Lavaud P, Andre F. Strategies to overcome trastuzumab resistance in HER2-overexpressing breast cancers: focus on new data from clinical trials. BMC Med. 2014;12:132. doi:10.1186/s12916-014-0141-2
OncoTargets and Therapy

\section{Publish your work in this journal}

OncoTargets and Therapy is an international, peer-reviewed, open access journal focusing on the pathological basis of all cancers, potential targets for therapy and treatment protocols employed to improve the management of cancer patients. The journal also focuses on the impact of management programs and new therapeutic agents and protocols on

\section{Dovepress}

patient perspectives such as quality of life, adherence and satisfaction. The manuscript management system is completely online and includes a very quick and fair peer-review system, which is all easy to use. Visit http://www.dovepress.com/testimonials.php to read real quotes from published authors. 